\title{
THERAPEUTIC PROFILING OF NANO ENCAPSULATED DIOSGENIN VIA ATTENUATING HORMONAL STATUS, CELL PROLIFERATION, INFLAMMATORY RESPONSES, AND APOPTOSIS IN AN ANIMAL MODEL OF MAMMARY ONCOGENESIS
}

\author{
MANOBHARATHI VENGAIMARAN, KALAIYARASI DHAMODHARAN, MIRUNALINI SANKARAN*
}

Department of Biochemistry and Biotechnology, Faculty of Science, Annamalai University, Annamalainagar 608002, Tamil Nadu, India *Email: mirunasankar@gmail.com

Received: 28 Jul 2021, Revised and Accepted: 25 Aug 2021

\section{ABSTRACT}

Objective: The central motive of this study is to explore the therapeutic impact of Diosgenin encapsulated Chitosan nanoparticles (DG@CS-NP) on mammary carcinogenesis in female Sprague Dawley rats via modulating hormonal status, cell proliferation, inflammatory responses, and Apoptosis.

Methods: 7,12-dimethylbenz(a)anthracene (DMBA) was administered subcutaneously near the mammary gland (25 mg/kg b. wt) to provoke mammary tumor in female Sprague Dawley rats. Following the progress of a tumor, DMBA-induced tumor-bearing rats were medicated orally with 5 mg/kg b. wt of DG@CS-NP. Consequently, the expression of ER, PR, PCNA, Cyclin D1, NF- $\kappa B$, TNF- $\alpha$, Bcl-2, Caspases-3, and p53 in experimental rats were revealed via architectural immunohistochemistry. Further, Diosgenin interactions with these proteins were evidently confirmed by molecular docking analysis.

Results: As a result, we noticed diminished levels of ER, PR, PCNA, Cyclin D1, NF- $\kappa$ B, TNF- $\alpha$, and Bcl-2 expressions in DG@CS-NP medicated rats as well as with elevated levels of Caspases-3 and p53 expressions. In DMBA rats, the expressions were vice versa. Additionally, molecular docking analyses support these outcomes by highlighting the strong interaction between Diosgenin and breast cancer targets.

Conclusion: These reports prove that DG@CS-NP imposes its therapeutic impact by hormonal adjustments, downregulating proteins involved in inflammation and cellular proliferation, and thereby promotes apoptosis by impeding apoptotic inhibitors.

Keywords: Diosgenin, Mammary carcinogenesis, Hormonal status, Cell proliferation, Apoptosis, Molecular docking

(C) 2021 The Authors. Published by Innovare Academic Sciences Pvt Ltd. This is an open access article under the CC BY license (https://creativecommons.org/licenses/by/4.0/) DOI: https://dx.doi.org/10.22159/ijap.2021v13i6.42777. Journal homepage: https://innovareacademics.in/journals/index.php/ijap

\section{INTRODUCTION}

Cancer is a pioneering root of death and a notable hindrance in rising life expectancy worldwide. Among various types of cancer, in 2020 female breast cancer (BC) superseded with a record of 2.3 million additional diagnoses $(11.7 \%)$, followed by lung $(11.4 \%)$ colorectal $(10.0 \%)$, prostate $(7.3 \%)$, and stomach (5.6\%) cancers [1]. Although countless proven curative regimens prevail in BC management, which encompasses surgery, radiation and chemotherapy, they are really pricey and consequently seem to be counterproductive in certain circumstances via which cancer mortality continues to be climbed up [2]. This disease has been lethal in several instances due to its multifaceted origin and the vast variety of endogenous and exogenous stimuli which can trigger alternative pathways that lead to cellular proliferation [3]. As per emerging shreds of evidence, environmental toxins are arguably blamed for approximately $75 \%$ of all cancers. Humans often ingest environmental carcinogens such as polycyclic aromatic hydrocarbons (PAHs), which can arise from partial combustion of fossil fuels, cigarette emissions, diesel fumes, and use of chargrilled and smoked foods, barbequed meat, and burned up cooking oils. Aromatic carcinogenesis is notably detrimental to the breasts because it can be retained and dispersed in fat cells and thereby accounts for BC progressions [4]. A study on PAH-induced mammary carcinogenesis in experimental animals will shed light on the diversified molecular processes driving the abrupt transition of healthy cells to extremely malignant forms [5]. 7, 12-dimethylbenz (a) anthracene (DMBA), a kind of PAH and a well-known, sitespecific mammary carcinogen that's been utilized to provoke cancer in experimental rats. Animal studies with chemically mediated cancer development remain consistent and thus are ubiquitously applied during cancer research to uncover the underlying enigmatic molecular BC mechanisms such as elevated hormonal status, escalated cellular proliferation, aberrant inflammation, and dysregulated apoptosis [6].

Steroidal hormones such as estrogen and progesterone engage a prominent role in $\mathrm{BC}$ progression. The status of the Estrogen receptor (ER) and Progesterone receptor (PR) in BC is a key predictor for hormone response and disease prognosis [7]. As contrasted with malignant cells that either express any of such receptors (ER-/PR+, ER+/PR-) or neither of them (ER-/PR-), combined activation of both receptors $(\mathrm{ER}+/ \mathrm{PR}+)$ indicates fewer aggressive nature. Around $70 \%$ of human mammary cancers possess both hormone receptor-positive [8]. Beyond hormone receptors, BC pathogenesis is also implicated in cellular proliferation processes. Proliferating cell nuclear antigen (PCNA), an activator of DNA polymerase $\delta$, is found in all proliferating cells and performs an effective role in cell cycle supervision, DNA replication, repair, and epigenetic management. PCNA expression escalated incrementally when tissues advanced from normal epithelium to hyperplasia and dysplasia [9]. Cyclin D1, another primary cell cycle regulatory protein requisite for normal breast lobule-alveolar growth. Studies of selective exclusion of the cyclin D1 gene culminated in the poorer mammary gland growth and mitigating the progress of BC. Regrettably, elevated cyclin D1 expression has indeed been recorded in close to $50 \%$ of human $\mathrm{BC}$ [10]. In addition, cyclin D1 acts as an intervening agent in other cell cycle mechanisms, including Nuclear Factor Kappa-B (NF- $\kappa \mathrm{B})$. As it has been pinpointed as a core controller of countless inflammatory and carcinogenic processes. The NF- $\mathrm{KB}$ mechanisms are accountable for cell proliferation, metastasis, inflammation, invasion, angiogenesis, and differentiation in a multitude of cancers, specifically hormone-independent BC [11]. Consequently, TNF- $\alpha$ (Tumor Necrosis Factor-alpha) receptor activity was also blocked by activated NF- $\kappa B$ dimers as connected with the inhibitor of $\kappa \mathrm{B}(\mathrm{I} \kappa \mathrm{B})$ proteins. TNF- $\alpha$ is a multipurpose cytokine, performing a leading role in apoptosis, inflammation, and immunity. It has been shown to be enriched in the serum samples of patients with advanced-stage $\mathrm{BC}$, and it's been linked to an upswing in the amount and scale of metastatic BC [12]. Moreover, NF- $\kappa \mathrm{B}$ is also connected to both the repression of apoptosis and cell proliferation. NF- $\kappa B$ dysregulation affects the activity of cell deathregulating genes, contributing to the up-regulation of antiapoptotic and pro-survival genes, such as the members of the Bcl-2 
family [13]. However, p53, the first-ever tumor suppressor gene, operates to suppress and inhibit the proliferation of abnormal cells, blocking the progress of cancer. Silencing the p53 tumorsuppressor mechanism seems to be the most influential antiapoptotic lesion observed in cancers. It can trigger a multitude of actions particularly DNA repair, cell-cycle arrest, senescence, differentiation, and apoptosis [14]. An extensive insight into these detrimental molecular dysfunctions and their core signaling pathways opens the door to inventive therapeutic approaches.

Therefore, adopting a holistic curative strategy to cope with this fatal situation may be a valuable alternative, as it's evident that compounds arising via natural origins can combat the virulence of $\mathrm{BC}$, inhibit cancer cell proliferation, and alter cancer-related pathways [15]. In particular, Saponins from dietary origin gains recent attention by targeting a multitude of carcinogenic mechanisms, offering optimistic responses towards cancers and therefore serving an active part in treating cancer. Moreover, as a natural by-product, saponins appear to be a promising candidate for anticancer fundamental research [16]. Among that, Diosgenin (DG), a Phyto steroidal sapogenin, in fenugreek (Trigonella foenumgraecum) and wild yams (Dioscorea sp.), promises to be a fruitful bioactive chief compound of interest for both prevention and therapy of BC. Its anti-BC potency has been evidenced via modulating critical molecular candidates associated with growth, differentiation, inflammation, apoptosis, and oncogenesis [17]. Absurdly, DG has minimal oral bioavailability owing to its impoverished solubility/high lipophilicity, which becomes a crucial barrier for operating as a successful therapeutic agent. As a response, we formulate DG nanoparticles (NP's) using chitosan (CS) as an encapsulated carrier, culminating in improved bioavailability, strong drug payload, and controlled drug delivery at the target site. Therefore, we initiated an exploration to appraise the therapeutic prospects of DG@CS-NP on DMBA induced mammary carcinogenesis via regulating hormonal status, inflammatory response, as well as cell proliferation and apoptosis using an in vivo and in silico approach.

\section{MATERIALS AND METHODS}

\section{Chemicals}

Diosgenin, 7, 12-dimethylbenz(a)anthracene (DMBA), Chitosan, Sodium tripolyphosphate (TPP) were purchased from SigmaAldrich. Monoclonal antibodies such as ER, PR, Cyclin D1, PCNA, NF- $\kappa$ B, TNF- $\alpha$, Bcl-2, Caspase-3, and p53 were purchased from Santa Cruz Biotechnology, USA. All the other chemicals and reagents used were of analytical grade procured from nearby commercial sources.

\section{Preparation and characterization of DG@CS-NP}

DG@CS-NP, CS-NP, and Free DG@NP were synthesized by the ionic gelation method using TPP as a gelating agent. These lyophilized samples were further characterized by UV, SEM, FTIR, In vitro drug release, and pharmacokinetic study [18].

\section{Animals and diet}

Female Sprague Dawley rats weighing 130-150 g were purchased from Biogen Laboratory Animal Facility, Bangalore, India. Animals were kept in six spacious polypropylene cages and housed under normal laboratory conditions: temperature $\left(27 \pm 2{ }^{\circ} \mathrm{C}\right)$ and humidity $(55 \pm 5 \%)$ with a $12 \mathrm{~h}$ light/dark cycle and standard pellet diet and water ad libitum provided in the Central Animal House, Rajah Muthiah Medical College, and Hospital, Annamalai University. Rats were granted a whole week to adapt well before the experiment begins. The experimental approach was officially endorsed by Annamalai University's Institutional Animal Ethics Committee (IAEC) guided by the Committee for the Purpose of Control and Supervision of Experimental Animals (CPCSEA) (Registration number 160/PO/ReBi/S/1999/CPCSEA). (Proposal No. 1241 dated 23.04.2019). The animals were cared for in compliance with the Indian National Law on animal care and use by adhering to the norms and guidelines of Annamalai University's ethical committee for animal experimentation.

\section{Tumour induction}

DMBA has been used as a potent mammary chemical carcinogen for the current research. Mammary tumors were induced by a single subcutaneous injection of DMBA ( $25 \mathrm{mg} / \mathrm{kg}$ body weight) dissolved in a $1 \mathrm{ml}$ emulsion of sunflower oil $(0.75 \mathrm{ml})$ and physiological saline $(0.25 \mathrm{ml})$ for each rat [19].

\section{Experimental design}

The animals were randomly allocated into experimental and control groups and segmented into six groups comprising six animals each (fig. 1). Animals from Group I will be served as the control. During the first week of the experiment, Groups II-V will receive a single subcutaneous injection of $25 \mathrm{mg} / \mathrm{kg}$ b. wt DMBA. After $7 \mathrm{w}$, Groups III and IV will be medicated with DG and DG@CS-NP at $10 \mathrm{mg} / \mathrm{kg} \mathrm{b}$. wt and $5 \mathrm{mg} / \mathrm{kg} \mathrm{b}$. wt, respectively, thrice a week, orally. Groups V and VI will be supplied with CS-NP and free DG@NP at the concentrations of $5 \mathrm{mg} / \mathrm{kg}$ b. wt, thrice a week ( $21 \mathrm{~d}$ ), orally. The dosage applied in the current study is based on earlier analyses $[20,21]$. The experiment was terminated at the end of $14 \mathrm{w}$, and all the rats were sacrificed. The tissues were promptly dissected, washed thoroughly with ice-cold saline, and were preserved in $10 \%$ formalin, paraffin-embedded, partitioned, and fixed on polylysinecoated glass slides for immunohistochemical staining.

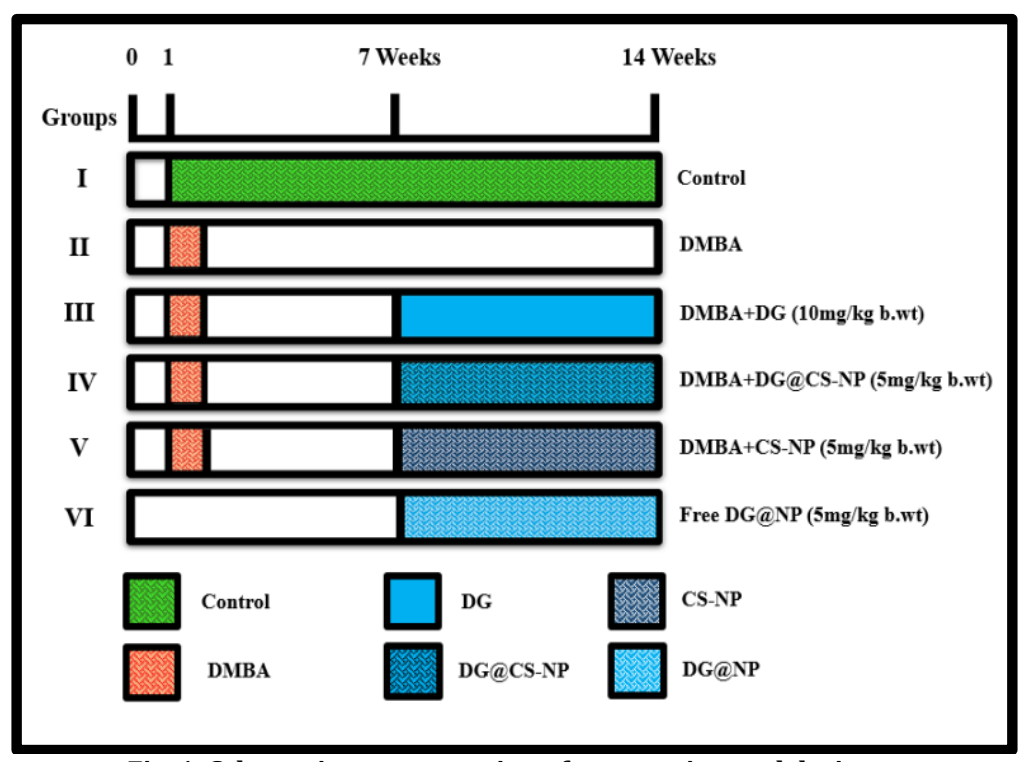

Fig. 1: Schematic representation of an experimental design 


\section{Immunohistochemistry}

The tissue sections were deparaffinized by heating them at $60{ }^{\circ} \mathrm{C}$ for $10 \mathrm{~min}$, accompanied by 3 xylene rinses. After been gradually hydrated with graded alcohol, the slides were then incubated in citrate buffer ( $\mathrm{pH}$ 6.0) in a microwave oven for two cycles of 5minute periods for antigen retrieval. After cooling for $20 \mathrm{~min}$, the sections were rinsed with Tris-buffered saline (TBS) and washed with $3 \% \mathrm{H}_{2} \mathrm{O}_{2}$ in Dis. $\mathrm{H}_{2} \mathrm{O}$ for 15 min to block endogenous peroxidase reaction. Through incubating the sections for $20 \mathrm{~min}$ with standard goat serum, non-specific antibody binding was minimized. The sections had been then incubated for 1 hour at Room Temperature (RT) with mouse monoclonal antibodies (ER, PR, Cyclin D1, PCNA, NF- $\kappa B$, TNF- $\alpha$, Bcl-2, Caspase-3, p53). The slides were rinsed with TBS before being incubated for $30 \mathrm{~min}$ at RT with anti-rabbit/mouse biotin-labeled secondary antibodies, backed up with streptavidin-biotin-peroxidase. The immune precipitate was stained with 3,3'-diaminobenzidine and counterstained with hematoxylin. The key antibody was substituted with TBS for negative controls. For positive controls, each antibody was run at the same time [22]

\section{Molecular docking}

Molecular docking analyses were performed using Auto dock software in the auto dock 4.2.6 package. The 3-D structures of the protein targets for $\mathrm{BC}$ were retrieved from the Protein Data Bank (PDB) using their PDB IDs, such as ER (2QR9), PR (1A28), CyclinD1 (2W96), PCNA (3VKX), Caspase-3 (3KJF), Bcl-2 (6QGG), NF-kB (1A3Q), TNF- $\alpha$ (7JRA), and p53 (4IBW). The DG structure used in this study was accessed from the PubChem compound database under the PubChem CID: 99474. After the protein and ligand preparation, the generation of receptor grids and the docking of ligands were also carried out [23]. The Biovia Discovery studio Visualizer was then used to explore the mode of binding by picturizing the 2D and $3 \mathrm{D}$ interactions and thereby examining such interactions among the multiple receptors and the ligand by reviewing all of the individual amino acid residues which interfered in binding.

\section{RESULTS}

\section{Immunohistochemical findings}

Fig. 2 shows the immunohistochemical expressions of ER, PR, Cyclin D1, PCNA, NF- $\kappa$ B, TNF- $\alpha$, Bcl-2, Caspase-3, p53 in the mammary tissues of both the control and experimental rats. The expressions of ER, PR, Cyclin D1, PCNA, NF- $\kappa$ B, TNF- $\alpha$, Bcl-2 were elevated in Group II and $V$ rats with ductal structures exposing noted focal necrosis and inflammations. Inversely, the ER, PR, Cyclin D1, PCNA, NF- $\kappa B$, TNF- $\alpha$, Bcl-2 expressions were diminished in Group III and IV, posing ductal structures with no evidence of inflammation fibrosis, and malignancy. Whereas Group I and VI shows normal expressions with zero evidence of inflammation. Conversely, the expressions of Caspase- 3 and p53 were found to be diminished in Group II and V rats. In addition, it embodies an altered increased expression in Group III and IV. The control rats Group I and VI exhibits normal expressions of Caspase-3 and p53. As an outcome, DG@CS-NP 5 $\mathrm{mg} / \mathrm{kg}$ b. wt was proven to be more active than DG $10 \mathrm{mg} / \mathrm{kg} \mathrm{b}$. wt in all scenarios.

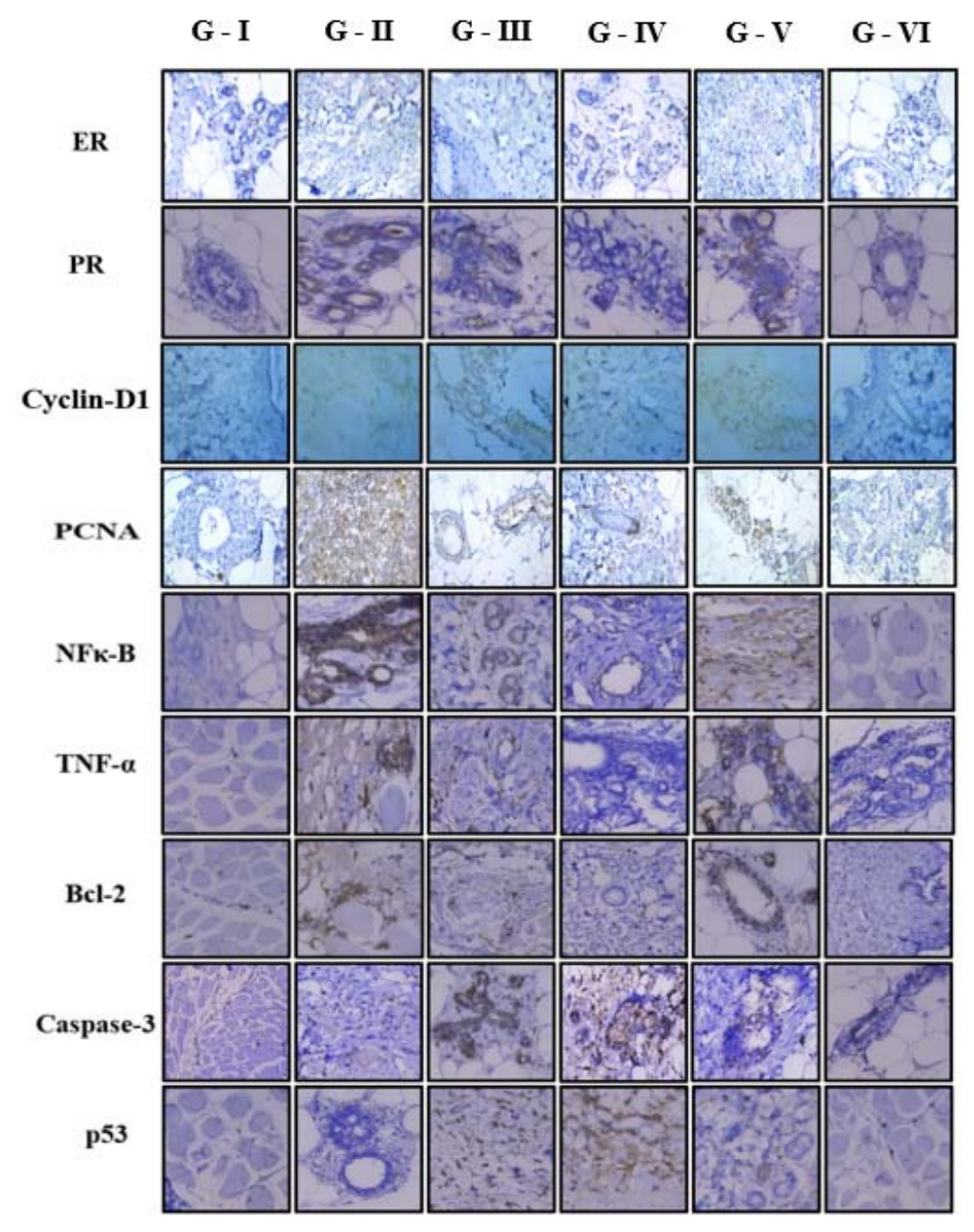

Fig. 2: Immunohistochemical expressions of ER, PR, Cyclin D1, PCNA, NF-KB, TNF- $\alpha$, Bcl-2, Caspase-3, p53 


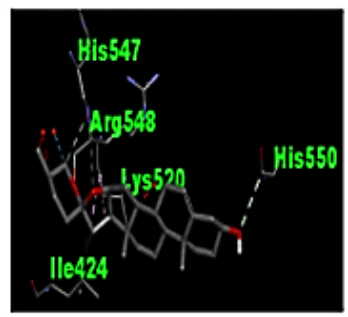

ER-DG

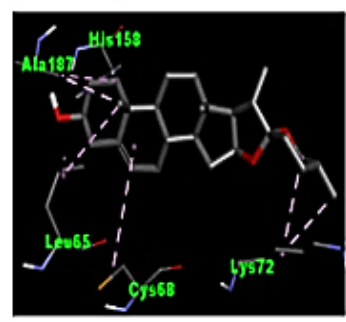

Cyclin-D1 -DG

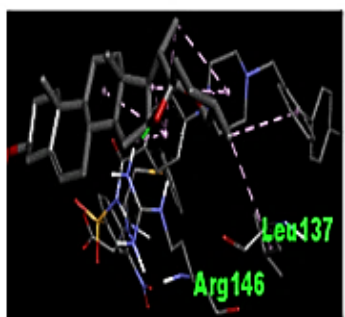

Bcl-2 -DG

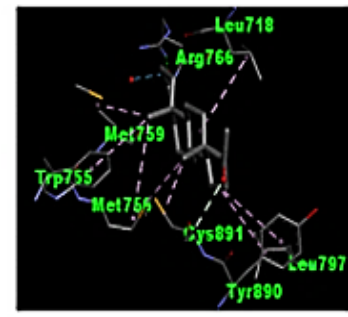

PR.DG

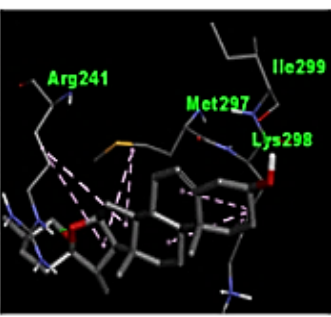

NF-kB -DG

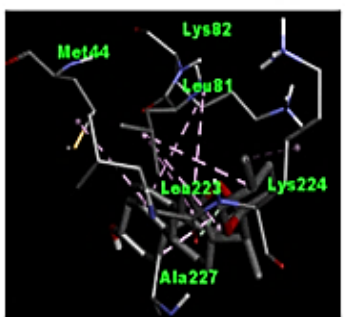

Caspase-3 -DG

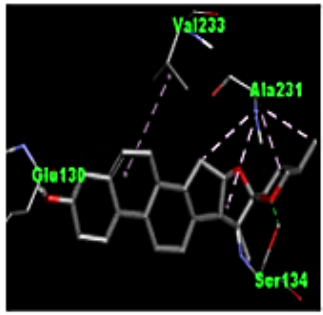

PCNA.DG

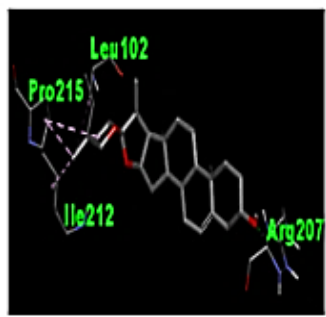

TNF-a -DG

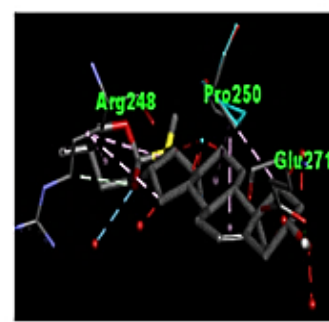

p53.DG

Fig. 3: 3-D interactions of diosgenin with active breast cancer targets
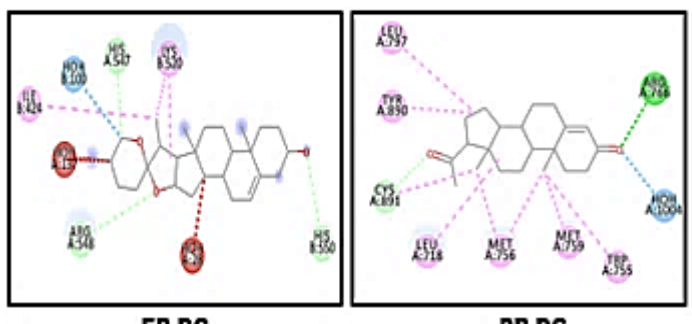

PR.DG

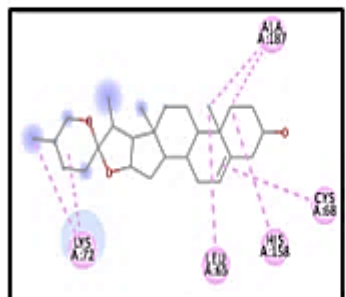

Cyclin-D1 -DG

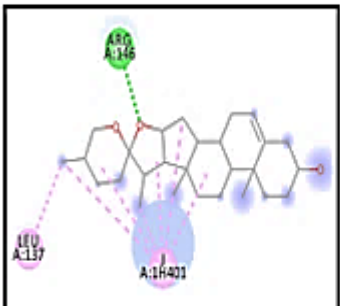

Bcl-2 -DG

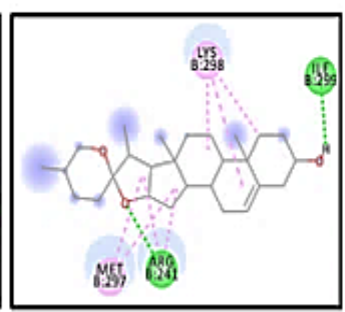

NF.kB -DG

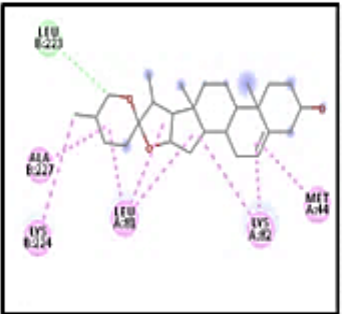

Caspase-3 -DG
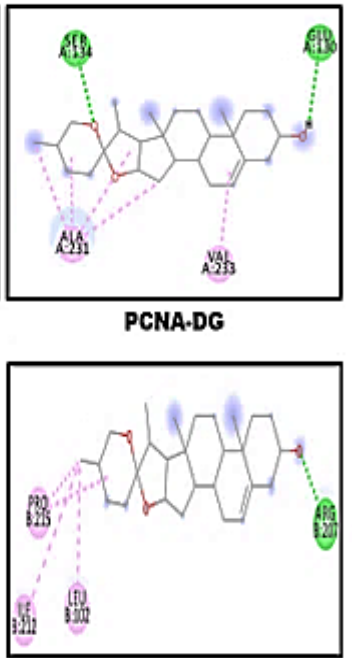

TNF- $a \cdot D G$

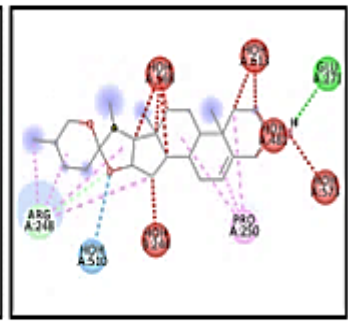

p53.DG

Fig. 4: 2-D interactions of diosgenin with active breast cancer targets 


\section{Molecular docking findings}

We employed bioinformatics approaches to uncover the binding sites of DG that validate its interactions with all these $\mathrm{BC}$ targeted proteins (ER, PR, Cyclin D1, PCNA, NF- $\kappa B$, TNF- $\alpha$, Bcl-2, Caspase-3, p53). Docking analyses proved that DG strongly interacts with these catalytic domains via a plethora of interacting core residues that sit in these active interactions with significant binding affinity. From the docking studies, we found that DG has the best binding scores with ER $(-9.95 \mathrm{kcal} / \mathrm{mol})$ with active residues Ile 424, Lys 520, His 547, Arg 548, His 550 and also shows good affinity with PR (-8.35 $\mathrm{kcal} / \mathrm{mol}$ ) with Leu 718, Trp 755, Met 756, Met 759, Arg 766, Leu 797, Tyr 890, Cys 891 as key participants. In the DG-Cyclin D1 complex, it poses-8.40 $\mathrm{kcal} / \mathrm{mol}$ binding energy with key residues Leu 65, Cys 68, Lys 72, His 158, Ala 187. Similarly, in DG-PCNA complex, it shows-8.73 kcal/mol binding energy with Glu 130, Ser 134, Ala 231, Val 233 as major participants. In DG-NF- $\kappa B$ interactions, it shows-8.30 $\mathrm{kcal} / \mathrm{mol}$ binding energy and the key residues interacted are Arg 241, Met 297, Lys 298, Ile 299. Thus, in complex with TNF- $\alpha$, DG shows $-8.52 \mathrm{kcal} / \mathrm{mol}$ binding energy with core residues Leu 102, Arg 207, Ile 212, Pro 215. Along with Bcl-2, DG shows-9.06 kcal/mol binding energy with Leu 137, Arg 146 interactive residues. DG-Caspase3 complex shows-8.65 kcal/mol binding energy with active residues Met 44, Leu 81, Lys 82 , Leu 223, Lys 224 , Ala 227. DG in complex with p53 possesses-7.79 kcal/mol as binding energy with Arg 248, Pro 250, Glu 271 as core participants. These data lend an approval for DG's active interactions with these BC targets, respectively (fig. 3 and fig. 4). Moreover, such outcomes are well congruent with immunohistochemical responses.

\section{DISCUSSION}

$\mathrm{BC}$ remains complicated to handle, as it holds an alarming statistical fatality in both advanced and emerging countries. Elevated BC threats offer a compelling rationale to pursue specific phytotherapeutics. Multiple empirical trials displayed an inverse interaction among the utilization of bioactive compounds and the emergence of BC [24]. DG is one such potent bioactive steroidal saponin that exudes multi-targeted anti-BC activity via abrogating major carcinogenic hotspots with zero toxicity [25]. Despite being a promising therapeutic candidate, its complete potency was restricted by its constrained physicochemical features like impoverished water solubility with diminished bioavailability. These obstacles hampered the DG's ability to hit its targeted spots. Currently, nano-sized controlled release formulation using biocompatible and biodegradable polymers is an emerging strategy for massive drug delivery and tumor targeting [18]. Therefore, to eradicate its countermeasures, a nanoscale drug delivery system affiliated with chitosan biopolymer as a drug carrier was planned to explore its ultimate therapeutic efficacy towards BC. As BC poses heterogenic nature with differentiated molecular and pathological attributes. Immunohistochemical (IHC) assessment affords a valuable predictive and prognostic knowledge of BC [22]. Additionally, Molecular docking, a sophisticated strategy used for probing a ligand's interaction with a target protein and is often intended to stipulate the binding aspects of therapeutics with specific target proteins for BC [27]. In this current study, DG@CS-NP halted the progress of rat mammary carcinoma by impeding inflammation, triggering apoptosis, and altering hormonal imbalances. Furthermore, it influences cell cycle progression by hindering cellular proliferation through down-regulating cyclin D1 and PCNA, with a rise in p53 immunohistochemical expression. In line with our results, Shishodia et al. reported that DG blocked TNF$\alpha$ induced transcription of NF- $\mathrm{KB}$ mediated gene products, thereby silencing inflammation, constraining proliferation, and promoting apoptosis [28].

Steroidal hormones hold paramount roles in the emergence and progression of $\mathrm{BC}$. Estrogen and progesterone functions were driven by nuclear receptors such as ER- $\alpha$ and ER- $\beta$ and PR-A and PR-B, which facilitate the multiplication of both healthy and cancerous mammary gland epithelial cells by modifying intracellular signaling [29]. According to the American Society of Clinical Oncology/College of American Pathologists (ASCO/CAP) regulations, ER and PR might be labeled positive when $1 \%$ or more of cancerous cells show nuclear staining of any intensity [30]. Therefore, in our results, DG@CS-NP proves its therapeutic potency by effectively normalizing the imbalanced hormone levels to a near-normal level. Furthermore, our findings endorsed prior with observations by Isabella et al., who hypothesized that the DIM@CS-NP in DMBA-induced rat mammary carcinoma embodies modifications in the ER and PR status of mammary tissues. Additionally, the binding efficacy of DG is also evident in its interactive potency with ER and PR [19].

Cellular proliferation ratios reveal significant insights about tumor progression and aggression in BC. Proliferation assessment is highly tied up with the tumor burden; thereby, it has been exploited as a prognostic predictor in BC. Cellular proliferation is split up into divergent segments. At the first gap (G1) stage, cells in the dormant (G0) phase were triggered to enroll into an active cycle. The cell guides for DNA synthesis (the $\mathrm{S}$ phase) at this timeframe, which is accompanied by a subsequent stage of relative inactivity (G2) and prepping for chromatid segregation in the mitotic (M) phase [31]. Cyclin D1 and PCNA are key players in the proliferation of BC cells. As Cyclin D1 is a pivotal controller of the cell cycle's G1 phase. Cell cycle halt arises when cyclin D1 activity was blocked, whereas unrestrained overexpression of the protein drives G1. Cyclin D1 coupling with PCNA strongly suppresses DNA synthesis. Clearly, our findings reveal that DMBA induced tumor-bearing rats displayed higher concentrations of PCNA and Cyclin D1, signaling accelerated cellular proliferation. Furthermore, DG@CS-NP medicated rats proved a fall in PCNA and Cyclin D1 expression, indicating diminished proliferative activity. Additionally, the interaction between DG and cell proliferative targets also shows significant interaction with the active participation of multiple key interactive residues.

Inflammatory microenvironment has increasingly emerged as a hot topic in the $\mathrm{BC}$ domain. In the linkage between inflammation and cancer initiation, TNF- $\alpha$ promotes carcinogenic processes. TNF- $\alpha$ latches with two receptors, the omnipresent TNF- $\alpha$ receptor 1 (TNFR1) and the hematopoietically confined TNFR2, and thus alters a signaling loop that triggers transcriptional regulators, which are valuable in cancer pathogenesis, including cell growth, migration, vasculature, and immune surveillance dysfunction. Additionally, NF$\kappa B$ response with TNFR-associated death domain protein (TRADD) stimulation and ultimately promotes cell proliferation. TNFR1 activation promotes both canonical and non-canonical NF- $\mathrm{kB}-$ dependent transcriptional gene regulators which code for prosurvival and pro-inflammatory mediators [32]. Therefore, evidently, DG@CS-NP-administered rats depict declined inflammatory expressions when compared to DMBA induced rats which express an elevated level of TNF- $\alpha$ and NF- $\kappa$ B expressions. In addition to this, DG also proves its strong binding ability with such inflammatory protein targets.

Apoptotic apparatus is driven by an alteration in the proportion of anti and pro-apoptotic proteins. Bcl-2, a well-known anti-apoptotic protein, blocks the release of pro-apoptotic proteins from mitochondria via a multitude of strategies, notably conserving mitochondrial membrane integrity and attaching to proapoptotic participants of the Bcl-2 family. It can rescue cells from apoptotic cell death by halting caspase-3-dependent proteolytic cascades. Consequently, the tumor suppressor protein p53 operates as a transcriptional modulator genomic protector, blocker of cancer progression, and stimulator of apoptosis. p53 promotes apoptosis by permeabilizing the mitochondrial outer membrane and creating complexes with Bcl-2 proteins, culminating in cytochrome-c release [33]. As per the above discussion, DG@CS-NP-treated rat's exhibit declined $\mathrm{Bcl}-2$ protein expression and rising Caspase- 3 expression via enhanced p53 activation. As an outcome, cancer progression is halted by promoting apoptotic cell death. In contrast, DMBA-injected rats had greater Bcl-2 expression with lesser Caspase-3 and p53 expression (fig. 5). These influencing capacities of DG were further supported by its stubborn binding affinity with these key target proteins. 


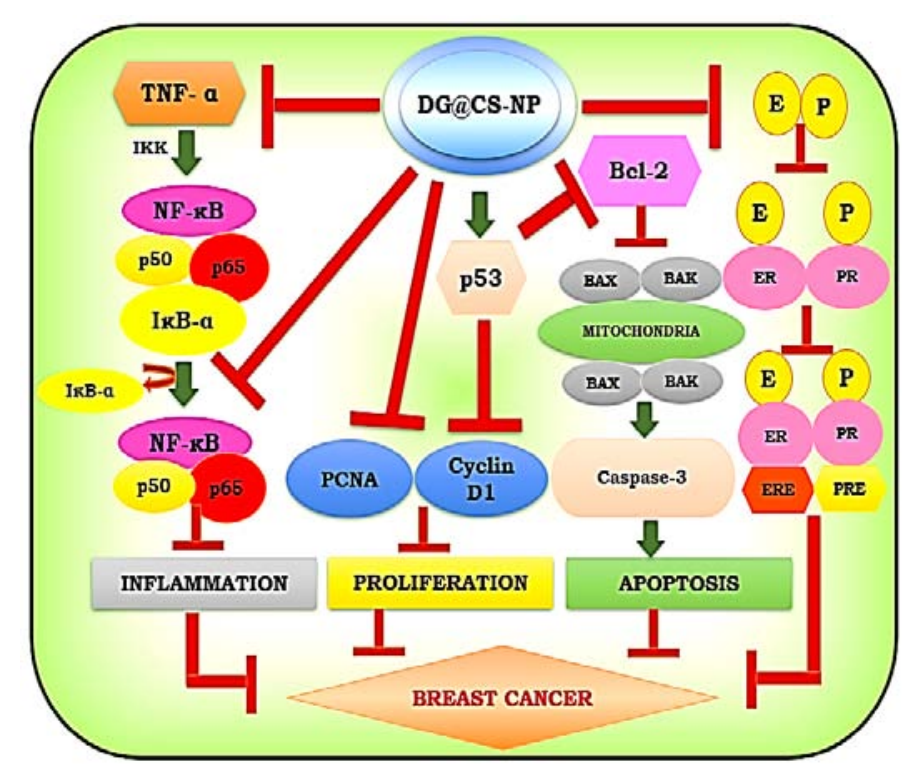

Fig. 5: Overall therapeutic mechanism of DG@CS-NP on rat mammary carcinoma

\section{CONCLUSION}

In conclusion, the entire analysis strongly validates the antiproliferative, anti-inflammatory, and apoptotic impacts of DG@CS-NP in breast cancer therapy via downregulating ER, PR, cyclin-D1, PCNA, TNF- $\alpha$, NF- $\kappa B$, and Bcl-2 expressions along with the upregulation of Caspase- 3 and p53 expressions. Both immunohistochemical expressions and molecular docking provide convincing proof that DG@CS-NP holds remarkable therapeutic potentials thereby it acts as a promising breast cancer chemotherapeutic candidate.

\section{ACKNOWLEDGMENT}

The authors would like to thank the Department of Biochemistry and Biotechnology, Annamalai University for providing the research facilities and support. The authors would also like to thank Central Animal House, Rajah Muthiah Medical College, and Hospital, Annamalai University for animal maintenance facilities.

\section{FUNDING}

Nil

\section{AUTHORS CONTRIBUTIONS}

All the authors have contributed equally.

\section{CONFLICT OF INTERESTS}

\section{Declared none}

\section{REFERENCES}

1. Sung H, Ferlay J, Siegel RL, Laversanne M, Soerjomataram I, Jemal A, Bray F. Global cancer statistics 2020: GLOBOCAN estimates of incidence and mortality worldwide for 36 cancers in 185 countries. CA Cancer J Clin. 2021;71(3):209-49. doi: 10.3322/caac.21660, PMID 33538338.

2. Manobharathi V, Kalaiyarasi D, Mirunalini S. A concise critique on breast cancer: a historical and scientific perspective. Res J Biotech. 2021;16(7):220-30. doi: 10.25303/167rjbt22021.

3. Perez Soto E, Estanislao Gomez CC, Perez Ishiwara DG, Ramirez Celis C, del Consuelo Gomez Garcia M. Cytotoxic effect and mechanisms from some plant-derived compounds in breast cancer. In: Cytotoxicity 2019;6:1-24.

4. Korsh J, Shen A, Aliano K, Davenport T. Polycyclic aromatic hydrocarbons and breast cancer: a review of the literature. Breast Care (Basel). 2015;10(5):316-8. doi: 10.1159/ 000436956 , PMID 26688678.

5. Vinothini G, Murugan RS, Nagini S. Evaluation of molecular markers in a rat model of mammary carcinogenesis. Oncol Res.
2009;17(10):483-93. doi: 10.3727/096504009789735459, PMID 19725228.

6. Karimi B, Ashrafi M, Shomali T, Yektaseresht A. Therapeutic effect of simvastatin on DMBA-induced breast cancer in mice. Fundam Clin Pharmacol. 2019;33(1):84-93. doi: 10.1111/fcp.12397, PMID 29962034.

7. Port Louis LR, Varshney KC, Nair MG. An immunohistochemical study on the expression of sex steroid receptors in canine mammary tumors. Int Sch Res Not. 2012;1:1-7.

8. Alvarado A, Lopes AC, Faustino-Rocha AI, Cabrita AMS, Ferreira R, Oliveira PA, Colaço B. Prognostic factors in MNU and DMBAinduced mammary tumors in female rats. Pathol Res Pract. 2017;213(5):441-6. doi: 10.1016/j.prp.2017.02.014, PMID 28285967.

9. Vinothkumar V, Manoharan S, Sindhu G, Nirmal MR, Vetrichelvi V. Geraniol modulates cell proliferation, apoptosis, inflammation, and angiogenesis during 7, 12-dimethylbenz [a] anthracene-induced hamster buccal pouch carcinogenesis. Mol Cell Biochem. 2012;369(1-2):17-25. doi: 10.1007/s11010-0121364-1, PMID 22729742.

10. Lengare PV, Sinai Khandeparkar SG, Joshi AR, Gogate BP, Solanke SG, Gore SH. Immunohistochemical expression of cyclin D1 in invasive breast carcinoma and its correlation with clinicopathological parameters. Indian J Pathol Microbiol. 2020;63(3):376-81. doi: 10.4103/IJPM.IJPM_106_20, PMID 32769325.

11. Wang W, Nag SA, Zhang R. Targeting the NFkB signaling pathways for breast cancer prevention and therapy. Curr Med Chem. 2015;22(2):264-89. 10.2174/0929867321666141106124315, PMID 25386819.

12. Zhou XL, Fan W, Yang G, Yu MX. The clinical significance of PR, ER, NF- $\kappa$ B, and TNF- $\alpha$ in breast cancer. Dis Markers. 2014;2014:494581. doi: 10.1155/2014/494581. PMID 24864130.

13. Luo JL, Kamata H, Karin M. IKK/NF-kappaB signaling: balancing life and death--a new approach to cancer therapy. JClin Invest. 2005;115(10):2625-32. doi: 10.1172/JCI26322, PMID 16200195.

14. Purushothaman A, Nandhakumar E, Shanthi P, Sachidanandam TP. Antiproliferative and apoptotic effects of Shemamruthaa, a herbal preparation 7, 12-dimethylbenz (a) anthracene-induced breast cancer rats. J Evid Based Complementary Altern Med 2015;20:25968. doi: 10.1177/2156587215580434, PMID 25888591

15. Mitra S, Dash R. Natural products for the management and prevention of breast cancer. Evid Based Complement Alternat Med 2018;1:1-23. DOI: 10.1155/2018/8324696, PMID 29681985

16. Xu XH, Li T, Fong CM, Chen X, Chen XJ, Wang YT, Huang MQ Lu JJ. Saponins from Chinese medicines as anticancer agents. Molecules. 2016;21(10):1-27. doi: 10.3390/molecules21101326, PMID 27782048. 
17. Manobharathi V, Mirunalini S. Pharmacological characteristics of a phyto steroidal food saponin: Diosgenin. Afr J Biol Sci. 2020;2:77. doi: 10.33472/AFJBS.2.2.2020.77-87.

18. Arulmozhi V, Pandian K, Mirunalini S. Ellagic acid encapsulated chitosan nanoparticles for drug delivery system in human oral cancer cell line (KB). Colloids Surf B Biointerfaces. 2013;110:313-20. doi: 10.1016/j.colsurfb.2013.03.039, PMID 23732810.

19. Isabella S, Mirunalini S, Pandiyan K. 3,3'-Diindolylmethane Encapsulated Chitosan Nanoparticles Accelerates Inflammatory Markers, ER/PR, Glycoprotein and Mast Cells Population During Chemical Carcinogen Induced Mammary Cancer in Rats. Indian J Clin Biochem. 2018;33(4):397-405. doi: 10.1007/ s12291-017-0701-2, PMID 30319185.

20. Jagadeesan J, Nandakumar N, Rengarajan T, Balasubramanian MP. Diosgenin, a steroidal saponin, exhibits anticancer activity by attenuating lipid peroxidation via enhancing antioxidant defense system during NMU-induced breast carcinoma. J Environ Pathol Toxicol Oncol. 2012;31(2):121-9. doi: 10.1615/JEnvironPatholToxicolOncol.v31.i2.40.

21. Kumar BNP, Puvvada N, Rajput S, Sarkar S, Das SK, Emdad L, Sarkar D, Venkatesan P, Pal I, Dey G, Konar S, Brunt KR, Rao RR, Mazumdar A, Kundu SC, Pathak A, Fisher PB, Mandal M. Sequential release of drugs from hollow manganese ferrite nanocarriers for breast cancer therapy. J Mater Chem B. 2015;3(1):90-101. doi: 10.1039/c4tb01098a, PMID 32261929.

22. Isabella S, Mirunalini S. 3, 3'-diindolylmethane-encapsulated chitosan nanoparticles accelerate molecular events during chemical carcinogen-induced mammary cancer in Sprague Dawley rats. Breast Cancer. 2019;26(4):499-509. doi: 10.1007/s12282-019-00950-x, PMID 30684233

23. Morris GM, Goodsell DS, Pique ME, Lindstrom WL, Huey R, Forli S. AutoDock 4.2 user guide. Scripps Research Institute; 2009. p. $1-66$.

24. Younas M, Hano C, Giglioli-Guivarc'h N, Abbasi BH. Mechanistic evaluation of phytochemicals in breast cancer remedy: current understanding and future perspectives. RSC Adv. 2018;8(52):29714-44. doi: 10.1039/C8RA04879G.
25. Sethi G, Shanmugam MK, Warrier S, Merarchi M, Arfuso F, Kumar AP, Bishayee A. Pro-apoptotic and anti-cancer properties of diosgenin: a comprehensive and critical review. Nutrients. 2018;10(5):1-12. doi: 10.3390/nu10050645, PMID 29783752.

26. Kalaiyarasi D, Manobharathi V, Mirunalini S. Development of nano drugs: A promising avenue for cancer treatment. Res J Biotechnol. 2021;16:234-44.

27. Ferreira LG, Dos Santos RN, Oliva G, Andricopulo AD. Molecular docking and structure-based drug design strategies. Molecules. 2015;20(7):13384-421. doi: 10.3390/molecules200713384, PMID 26205061.

28. Shishodia S, Aggarwal BB. Diosgenin inhibits osteoclastogenesis, invasion, and proliferation through the

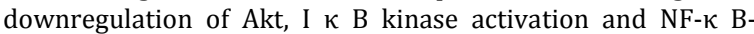
regulated gene expression. Oncogene. 2006;25(10):1463-73. doi: 10.1038/sj.onc.1209194, PMID 16331273.

29. Thangarasu R, Pachaiappan P, Subbaiyan T. Anti-estrogenic and anti-cell proliferative effect of allyl isothiocyanate in chemoprevention of chemically induced mammary carcinogenesis in rats. Pathol Oncol Res. 2020;26(2):913-25. doi: 10.1007/s12253-019-00638-9, PMID 30895454.

30. Tang P, Tse GM. Immunohistochemical surrogates for molecular classification of breast carcinoma: a 2015 update. Arch Pathol Lab Med. 2016;140(8):806-14. doi: 10.5858/arpa.2015-0133-RA, PMID 27472239.

31. Beresford MJ, Wilson GD, Makris A. Measuring proliferation in breast cancer: practicalities and applications. Breast Cancer Res. 2006;8(6):216. doi: 10.1186/bcr1618, PMID 17164010.

32. Martins GR, Gelaleti GB, Moschetta MG, Maschio-Signorini LB, Zuccari DA. Proinflammatory and anti-inflammatory cytokines mediated by NF- $\mathrm{\kappa B}$ factor as prognostic markers in mammary tumors. Mediators Inflamm. 2016;2016:9512743. doi: 10.1155/2016/9512743, PMID 26989335.

33. Kumaraguruparan R, Prathiba D, Nagini S. Of humans and canines: Immunohistochemical analysis of PCNA, Bcl-2, p53, cytokeratin and ER in mammary tumours. Res Vet Sci. 2006;81(2):218-24. doi: 10.1016/j.rvsc.2005.08.002, PMID 16740286. 